\title{
The Effectiveness of CBTAC Protocol on Burdens of Alzheimer's Disease on Caregivers and their Sense of Coherence
}

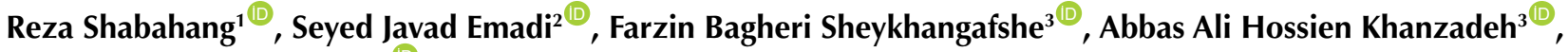 \\ Seyedeh Maryam Mousavi ${ }^{* * \mathbb{C}}$
}

1Department of Psychology, Faculty of Psychology and Educational Sciences, University of Tehran, Tehran, Iran ${ }^{2}$ Department of Clinical and Health Psychology, Faculty of Education and Psychology, Shahid Beheshti University, Tehran, Iran

${ }^{3}$ Department of Psychology, Faculty of Literature and Humanities, University of Guilan, Rasht, Iran

${ }^{4}$ Department of Nursing and Midwifery, Rasht Branch, Islamic Azad University, Rasht, Iran

\begin{abstract}
Background: Alzheimer's disease is predicted to increase dramatically in the near future. Alzheimer's caregiving brings about severe problems for caregivers. Considering the corrosive consequences of Alzheimer's disease on Alzheimer's caregivers, finding an effective intervention is necessary. Thus, the present research conducted by the aim of investigating the effectiveness of cognitive-behavioral treatment for Alzheimer's disease patients and their caregiver's protocol on burdens of Alzheimer's disease on caregivers and their sense of coherence (SOC).

Methods: This study was a quasi-experimental research with a pretest-posttest control group design. The target population included all mild Alzheimer's disease patients and their caregivers referred to the neurology ward of the hospitals in Rasht during summer 2018. According to the medical records and results of screening, 40 mild Alzheimer's disease, patients and their caregivers recruited by convenience sampling method. After sampling, research participants randomly assigned into two experimental $(\mathrm{n}=$ 20) and waitlist control $(n=20)$ groups. The Impact of Alzheimer's Disease on Caregiver Questionnaire and SOC scale were used to measure the burdens of Alzheimer's disease on caregivers and caregivers' SOC. The CBTAC provided for the experimental group participants in 25 sessions of 90-minutes. In the end, the data analyze has done by multivariate analysis of covariance (MANCOVA) and using SPSS24 software.

Results: The findings indicated that the CBTAC had a significant effect on the burdens and SOC of Alzheimer's caregivers in the experimental group $(P<0.01)$. In other words, the CBTAC decreases burdens and increase the SOC of Alzheimer's caregivers significantly $(P<0.01)$.

Conclusion: Based on the research results, the CBTAC is an effective intervention in decreasing caregiving burden and the increasing SOC of Alzheimer's caregivers. Therefore, CBTAC is a multicomponent intervention that can be used to improve the competency and mental health of Alzheimer's caregivers. Keywords: Alzheimer Caregiving; CBTAC Protocol; Burdens of Alzheimer's disease; Sense of Coherence.
\end{abstract}

\author{
*Correspondence to \\ Seyedeh Maryam Mousavi, \\ $\mathrm{PhD}$ in Health Psychology, \\ Department of Nursing and \\ Midwifery, Rasht Branch, \\ Islamic Azad University, \\ Rasht, Iran. \\ Email: \\ mmousavi@iaurasht.ac.ir
}

Published online March 10, 2020

Citation: Shabahang R, Emadi SJ, Bagheri Sheykhangafshe F, Hossien Khanzadeh AA, Mousavi SM. The effectiveness of CBTAC protocol on burdens of Alzheimer's disease on caregivers and their sense of Coherence. Int Clin Neurosci J. 2020;7(2):81-87. doi:10.34172/ icnj.2020.06.

\section{Introduction}

Dementia is one of the most common neurological diseases and one of the most critical health-threatening factors in the elderly that has a devastating effect. ${ }^{1}$ Alzheimer's disease is one of the most prominent causes of dementia, with its prevalence increasing as the world's aging population grows. ${ }^{2}$ Alzheimer's disease is a brainrelated structural and functional disorder that gradually disrupts one's mental abilities such as thinking, reasoning, judgment, and memory ${ }^{3}$ and has symptoms such as memory problems, personality changes, depression, and anxiety. ${ }^{4}$ In other words, Alzheimer's disease recognized as a progressive amnestic disorder with associated neurological, cognitive, and behavioral deficits. ${ }^{5}$ In this disease, an abnormal accumulation of a protein called amyloid-beta occurs outside of the nerve cells, and Tao protein occurs inside the nerve cell. This protein accumulation inside and outside the nerve cell disrupts neuronal network communication and eventually destroys nerve cells. ${ }^{6,7}$ Atrophy of brain areas such as the hippocampus, amygdala, temporal lobe, and reduction of cortical thickness are other issues that have raised 
concerning Alzheimer's disease. ${ }^{8}$

In general, the multiplicity and variety of personal, family, and social problems and consequences of Alzheimer's disease complicate the care of patients. In addition to the harmful physical effects, the selfdestructive and gradual destruction of the patient's mind creates a wide range of physical, emotional, psychological, social, and economic needs for the affected person, family, and caregiver. Indeed, it must acknowledge that caring for patients with Alzheimer's goes far beyond a set of physically focused medical and nursing guidelines and is also a psychological and social phenomenon. ${ }^{9-11}$ Taking care of the elderly with Alzheimer's disease is a daunting task, and caregivers of this group of elderly faced with many problems such as stress, anxiety, and depression for proper care of the patient. ${ }^{12}$ The caring of the elderly with Alzheimer's disease, given the burden placed on caregivers, leads to psychological stress on caregivers. ${ }^{10}$ In other words, caring for this group of elderly affects the various dimensions of caregivers known as the concept of burdens of caregiver. ${ }^{10,13}$ In general, the burden of caring refers to the negative consequences of caring for chronic patients for their caregivers. ${ }^{14}$ Meanwhile, Alzheimer's elderly are among the groups that can bring high levels of caregiver burden to their caregivers. ${ }^{15}$ Ferrara et $\mathrm{al}^{12}$ report that stress, anxiety, and depression are common in Alzheimer's caregivers. The results of the study conducted by Montgomery et $\mathrm{al}^{16}$ show that Alzheimer's disease affects the mental health of patients and their caregivers. Sadik and Wilcock ${ }^{17}$ also emphasize the significant burden that Alzheimer's places on caregivers and the health care system. Also, Rymer et $\mathrm{al}^{18}$ confirm that caregivers of Alzheimer's patients experience a burden of caring. In another study, Pudelewicz et $\mathrm{al}^{10}$ point out the importance of caregiver burden and caregiver burnout. Cross-sectional and longitudinal results of the study conducted by Mohamed et $\mathrm{al}^{19}$ also show that the burden of care for Alzheimer's disease is an important issue that can be affected by psychiatric symptoms, behavioral disturbances and quality of patient's life. Moreover, the findings of studies by Brodaty et $\mathrm{al}^{20}$ and Etters et $\mathrm{al}^{21}$ show that caring for dementia and Alzheimer's patients is associated with adverse effects on caregivers' health. Studies show that caregiving burden in Alzheimer's disease is a critical issue that necessitates interventions to reduce the burden of caregivers' care in this group of elderly.

In addition to the burden of care, a sense of cohesion is another important issue for caregivers of Alzheimer's patients. Antonovsky ${ }^{22,23}$ put forward the concept of sense of coherence to explain the attitude of people to the stressors. Within the frame of the salutogenic model, Antonovsky introduced generalized resistance resources (GRR), in which repeated use of GRR leads to a sense of coherence (SOC). GRR, and SOC interrelated. The sense of coherence refers to a general tendency that sees the world and the environment of personal life as understandable, manageable, and meaningful, claiming that the way people view their lives has a positive impact on their health. ${ }^{22,23}$ The SOC is a health冈promoting resource that is related to the different aspects of health. ${ }^{24}$ Studies have shown the important role of the SOC in Alzheimer's care. Andrén and Elmståhl ${ }^{25}$ suggest that there is a relationship between the burden of care, perceived health, and the SOC of caregivers of dementia and Alzheimer's patients. Pretorius et al, ${ }^{26}$ Potgieter and Heyns, ${ }^{27}$ and Elnasseh et al ${ }^{28}$ also confirm the relationship between Alzheimer's care and a SOC. Furthermore, Välimäki et $\mathrm{al}^{29}$ examined the SOC in caregivers of Alzheimer's patients in the three-year follow-up. The results suggest a decrease in caregivers' SOC. Childers ${ }^{30}$ confirms the role of the SOC in health, health-related quality of life, and stress of Alzheimer's caregivers. Accordingly, the SOC is a very prominent component to consider Alzheimer's caregiver.

Alzheimer's disease is predicted to increase dramatically in the future, ${ }^{31}$ given the association of Alzheimer with anxiety, ${ }^{32}$ depression, ${ }^{33}$ personality disorders, ${ }^{34}$ sleep disorders, ${ }^{35}$ physical and functional health problems ${ }^{36}$ as well as negative family, ${ }^{37}$ social and economic consequences $^{38,39}$ of Alzheimer's disease, it is essential to find interventions related to Alzheimer's disease. In the meantime, psychological programs are among the interventions that have been proposed concerning Alzheimer and have considered in recent years. ${ }^{40}$

One of the psychological interventions for mild Alzheimer's disease is the cognitive-behavioral treatment for Alzheimer's disease patients and their caregivers. ${ }^{41}$ CBTAC is an intervention that incorporates specific cognitive (e.g., cognitive reconstructing) and behavioral (e.g., behavioral management) components and generally based on cognitive-behavioral theory. ${ }^{42}$ CBTAC consists of diagnosis and goal setting, psycho-education, engagement in pleasant activities, cognitive restructuring, life review, behavioral management techniques, and couples counseling and targets caregivers in addition to Alzheimer's patients. ${ }^{41}$ CBTAC can be an effective method of incorporating a wide range of cognitive and behavioral elements in both Alzheimer's patients and their caregivers, which can affect the burden of care and the SOC of caregivers of Alzheimer's patients.

Overall, effective psychological interventions are necessary for the view of the widespread Alzheimer's disease and its wide-ranging negative consequences for patients and their caregivers at the individual, interpersonal, and collective levels. In the meantime, CBATC can be an effective intervention in the burden of care and a SOC for caregivers of Alzheimer's patients, with emphasis on the cognitive and behavioral elements of both patients and caregivers. In this regard, the purpose of the present study is to examine the effectiveness of CBTAC protocol on the burdens of Alzheimer's disease on caregivers and their SOC. 


\section{Methods}

This study was a quasi-experimental research with a pretest-posttest control group design. The main purpose of the study was to investigate the effectiveness of CBTAC protocol on the burdens of Alzheimer's disease on caregivers and their SOC. The target population included all mild Alzheimer's disease patients and their caregivers referred to the neurology ward of the hospitals in first and second districts of Rasht during summer 2018. According to the medical records and results of screening, 40 mild Alzheimer's disease, patients and their caregivers recruited by convenience sampling method. Inclusion criteria for Alzheimer's disease patients included diagnosis of mild Alzheimer's disease, absence of uncontrolled severe physical and psychological disorders, and not receiving other psychological interventions. In the case of caregivers of Alzheimer's disease patients, inclusion criteria consisted of caring mild Alzheimer's disease patients, lack of serious physical and psychological problems, and not receiving specific physical and psychological interventions. The criteria for exclusion include absence in more than two sessions, causing problems in the intervention process, reluctance to cooperate in the treatment sessions, and lack of personal satisfaction. Written informed consent obtained from each participant. After sampling, research participants (Alzheimer's patients and their caregivers) randomly assigned into two experimental $(\mathrm{n}=20)$ and waitlist control $(\mathrm{n}=20)$ groups. The Impact of Alzheimer's Disease on Caregiver Questionnaire (IADCQ) $)^{13}$ and SOC scale ${ }^{43}$ were used to measure the burdens of Alzheimer's disease on caregivers and caregivers' SOC. Before the intervention, caregivers responded to study measurements, then the participants (both patients and caregivers) in the experimental group underwent the cognitive-behavioral treatment for mild Alzheimer's patients and their caregivers ${ }^{41}$ in 25 sessions of 90-minutes (2 sessions per week). The content of CBTAC sessions has been presented in Table 1 summarily. After the intervention, a post-test executed on both the experimental group and the waitlist control group. Data analysis have done by multivariate analysis of covariance (MANCOVA) and using SPSS version 24 software At the end of the study, Due to ethical considerations, the intervention also offered to the waitlist control group.

\section{The Impact of Alzheimer's Disease on Caregiver Questionnaire}

The IADCQ is developed to measure the level of burden of Alzheimer's disease on caregivers. The IADCQ composed of 12 items that measure Alzheimer's disease caregiver's burden across emotional, physical, social, financial, sleep, and time aspects. The response and rating format for the IADCQ is a 5-point scale with anchor points being not at all equal to 0 , and extremely equal to 4 . The participant's score is between $0-48$. Cole et $\mathrm{al}^{13}$ reported adequate reliability and validity of the questionnaire. The IADCQ had a mean scale score of 21.6 and a standard deviation of 10.8. Findings showed that the IADCQ is unidimensional and covers an ideal range of burdens. In Cole et al's study ${ }^{13}$ internal consistency with a coefficient alpha

Table 1. The Summary of the CBTAC Protocol

\begin{tabular}{|c|c|c|}
\hline Topics & Session Content & Setting \\
\hline \multirow{2}{*}{ Diagnosis and goal setting } & Diagnostic investigation & Joint \\
\hline & Behavior analysis and goal setting & Joint \\
\hline \multirow[t]{2}{*}{ Psychoeducation } & Psychoeducation & Joint \\
\hline & Setting the stage for pleasant activities & Joint \\
\hline \multirow[t]{2}{*}{ Engagement in pleasant activities } & Planning of pleasant activities & Possibly without caregiver \\
\hline & Establishing regular activities & Joint \\
\hline \multirow{3}{*}{ Cognitive restructuring } & Setting the stage for cognitive restructuring & Joint \\
\hline & Challenging negative thoughts & Possibly without caregiver \\
\hline & Practicing helpful thoughts & Joint \\
\hline \multirow{4}{*}{ Life review } & Childhood & Joint \\
\hline & Adolescence & Joint \\
\hline & Young adulthood & Without caregiver \\
\hline & Older adulthood & Without caregiver \\
\hline \multirow{2}{*}{$\begin{array}{l}\text { Training caregiver in behavior management } \\
\text { techniques }\end{array}$} & Setting the stage for behavior management & Joint \\
\hline & Changing problem behavior & Joint - Possibly without the caregiver \\
\hline \multirow{3}{*}{ Interventions for the caregiver } & Stress management and emotion regulation & Only caregiver \\
\hline & Pleasant activities & Only caregiver \\
\hline & Social support & Only caregiver \\
\hline \multirow{3}{*}{ Couples counseling } & Setting the stage for couples counseling & Joint \\
\hline & Communication and joint problem-solving training & Joint \\
\hline & Acceptance and planning for the future & Joint \\
\hline Closing of therapy & Summary and reflection & Joint \\
\hline
\end{tabular}


and an intra-class correlation coefficient were 0.93 and 0.68 , respectively. Additionally, correlations between the IADCQ and the SF-12v2, PCS, and MCS scales indicate the appropriate validity of the IADCQ. In the present study, the IADCQ was internally consistent, as well ( $\alpha=$ $0.87)$.

\section{The Sense of Coherence Scale}

The SOC Scale is a 29-items and single-factor instrument for measuring the SOC. The purpose of the SOC is to measure the comprehensibility, manageability, and meaningfulness. The SOC has a 7-point Likert scale with response choices ranging from 1 to 7 . Thirteen of the items have reversed in scoring. The questionnaire yields a summed score with a range from 29 to 203. Higher scores express a higher level of SOC. According to Antonovsky's study $^{43}$, the SOC has appropriate psychometric properties. The SOC had a different mean score (117.0 to 152.6) and a standard deviation (0.8 to 36.4 ). In 26 studies using SOC29 , the Cronbach alpha measure of internal consistency has ranged from 0.82 to 0.95 .

Furthermore, the test-retest reliability of the SOC was satisfactory. The correlations between the SOC and measures of global orientation to oneself and one's environment, health, illness and wellbeing, stressors, and attitudes confirmed the appropriate validity of the scale. The systematic review of Eriksson and Lindström ${ }^{44}$ indicates that the SOC is a reliable, valid, and crossculturally applicable instrument. Alipour and Sharif ${ }^{45}$ also confirmed the excellent psychometric properties of the SOC in Iran. In the present research, the alpha reliability was 0.88 .

\section{Results}

The mean and standard deviation of the burdens of Alzheimer's disease and the SOC pre-test-post-test scores in the experimental and control groups presented in Table 2. In this table, the results of the Kolmogorov-Smirnov test reported in order to verify the normal distribution of variables in two groups. According to this table, the Z-statistic of the Kolmogorov-Smirnov test was not significant for all variables. Therefore, it can conclude that the distribution of variables is normal.
For investigating the effectiveness of CBTAC protocol on burdens of Alzheimer's disease on caregivers and their SOC, MANCOVA was used.

The results of Levene's test for investigating the homogeneity of dependent variables variance in groups showed that the variance of burdens of Alzheimer's disease $\left(\mathrm{F}_{1.38}=0.43, P=0.513\right)$ and SOC $\left(\mathrm{F}_{1.38}=2.70, P\right.$ $=0.108>0.05$ )is equal in groups. The results of the $\mathrm{M}$ Box test for checking the equality of the covariance matrix of dependent variables between the experimental and control groups also showed that the covariance matrix of dependent variables of two groups was equal (M $\mathrm{Box}=4.757, \mathrm{~F}=1.49, P=0.214>0.05)$. The significance level of the Box test is more significant than 0.05, and this assumption established. The results of chi-square and Bartlett's tests for sphericity or significance of the relationship between burdens of Alzheimer's disease and SOC showed that the relationship between them was significant $\left(P<0.05, \chi^{2}=33.94, \mathrm{df}=2\right)$. Another critical assumption of multivariate analysis of covariance is the homogeneity of regression coefficients.

It should be noted that the test of homogeneity of regression coefficients investigated through the interaction of dependent and independent variables (intervention method) in pre-test and post-test. The interaction of these pre-tests and post-tests with the independent variable was not significant and indicated the homogeneity of the regression slope, so this assumption established. Due to the assumptions of multivariate analysis of covariance, the use of this test will have permitted. Multivariate analysis of covariance performed in order to find out the differences between groups (Table 3 ).

According to Table 3, the results showed the effect of the independent variable on the dependent variables; in other words, the experimental and control groups had significant difference at least in one of the variables of burdens of Alzheimer's disease and SOC, that $56 \%$ of total variances of the experimental and control groups were due to the independent variable regarding the calculated effect size. The statistical power of the test is also equal to one, indicating the adequacy of the sample size. However, in order to determine which domains are significant, the univariate analysis of covariance was used in the

Table 2. Descriptive Indices of Study's Variables in Wait List Control and Experimental Groups

\begin{tabular}{|c|c|c|c|c|c|c|}
\hline Variables & & Groups & Mean & SD & K-S Z & $\boldsymbol{P}$ \\
\hline \multirow{4}{*}{$\begin{array}{l}\text { Burdens of Alzheimer's } \\
\text { disease }\end{array}$} & \multirow{2}{*}{ Pre-test } & Wait List Control Group & 21.05 & 8.24 & 0.137 & 0.055 \\
\hline & & Experimental Group & 21.55 & 8.73 & 0.123 & 0.063 \\
\hline & \multirow{2}{*}{ Post-test } & Wait List Control Group & 21.20 & 3.62 & 0.124 & 0.125 \\
\hline & & Experimental Group & 17.15 & 3.42 & 0.103 & 0.161 \\
\hline \multirow{4}{*}{ SOC } & \multirow{2}{*}{ Pre-test } & Wait List Control Group & 120.45 & 11.54 & 0.123 & 0.128 \\
\hline & & Experimental Group & 120.05 & 15.22 & 0.108 & 0.081 \\
\hline & \multirow{2}{*}{ Post-test } & Wait List Control Group & 120.10 & 7.44 & 0.124 & 0.124 \\
\hline & & Experimental Group & 129.55 & 12.34 & 0.105 & 0.094 \\
\hline
\end{tabular}

SOC, sense of coherence; K-S Z: Z-statistic of the Kolmogorov-Smirnov. 
Table 3. The Results of Multivariate Analysis of Covariance on Mean PostTest Scores

\begin{tabular}{lllll}
\hline Test & Value & F & $\boldsymbol{P}$ & Effect Value \\
\hline Pillai's effect & 0.365 & 10.05 & 0.001 & 0.365 \\
Wilks Lambda & 0.635 & 10.05 & 0.001 & 0.365 \\
Hotelling trace & 0.575 & 10.05 & 0.001 & 0.365 \\
Roy's largest root & 0.575 & 10.05 & 0.001 & 0.365 \\
\hline
\end{tabular}

MANCOVA. The results of which reported in Table 4.

According to Table 4, F statistic for burdens of Alzheimer's disease (12.88) and SOC (8.09) was significant at 0.01 levels. These findings indicate that there is a significant difference between groups in these variables. Also, according to the calculated effect size, $26 \%$ of Burdens of Alzheimer's Disease and 18\% of the SOC were due to the effect of the independent variable. Accordingly, it can note that the CBTAC protocol was active on the burdens of Alzheimer's disease on caregivers and their SOC.

\section{Discussion}

The present study aimed to study the effectiveness of the CBTAC protocol on the burdens of Alzheimer's disease on caregivers and their SOC. Based on the findings, CBTAC was valid on Burdens and the SOC of Alzheimer's caregivers. The results indicated that the CBTAC was effective and reduced the caregiving burden and increased the sense of cohesion of Alzheimer's caregivers.

Alzheimer's disease has many negative consequences for Alzheimer's patients and their caregivers as a progressive amnestic disorder, ${ }^{5}$ which leads to problems in the care of Alzheimer's patients. ${ }^{9-11}$ Burdens of caregiver and a SOC are among the most critical issues to consider in caring for Alzheimer's patients. Alzheimer's disease puts stress on caregivers ${ }^{10}$ and leads to stress, anxiety, and depression in caregivers. ${ }^{12}$ In line with this, the results of the studies of Ferrara et al, ${ }^{12}$ Montgomery et al, ${ }^{16}$ Sadik \& Wilcock, ${ }^{17}$ Rymer et al, ${ }^{18}$ Pudelewicz et al, ${ }^{10}$ Mohamed et al, ${ }^{19}$ Brodaty et $\mathrm{al}^{20}{ }^{20}$ Etters et $\mathrm{al}^{21}$ emphasize the burden of Alzheimer's disease care. Also, the SOC is another critical issue in the care of Alzheimer's patients. According to the results of studies by Andrén \& Elmståhl, ${ }^{25}$ Pretorius et $\mathrm{al}_{,}{ }^{26}$ Potgieter and Heyns, ${ }^{27}$ Elnasseh et $\mathrm{al},{ }^{28}$ Välimäki et $\mathrm{al}^{29}$ and Childers, ${ }^{30}$ SOC is a prominent factor in the care of Alzheimer's disease. It should acknowledge that the burden of care and the SOC of Alzheimer's caregivers are critical issues that require interventions to reduce the burden of care and increase the SOC in caregivers. In the meantime, CBTAC can affect the burden of care and a SOC for patient caregivers as a cognitive-behavioral intervention while encompassing a wide range of cognitive and behavioral elements in both Alzheimer's patients and their caregivers. The results of this study are in line with the findings of the study conducted by Frostmeyer et $\mathrm{al}^{41}$ confirming the positive impact of CBTAC protocol on caregivers of Alzheimer's patients.

In explaining this, it should acknowledge that CBTAC positively affects both Alzheimer's patients and caregivers of Alzheimer's patients, with emphasis on psychoeducation, pleasant activities, cognitive restructuring, life review, behavior management, and couples counseling. CBTAC gives information about Alzheimer's disease and its stages, causes, treatments, and consequences for both patients and caregivers. This intervention also addresses engagement in pleasant activities and life review. CBTAC also leads to schemachanging, cognitive reframing, challenging dysfunctional thoughts, finding alternative thoughts, increasing thought control through cognitive restructuring. This intervention also has positive behavioral consequences concerning behavioral management and behavioral reactions. CBTAC protocol positively affects the burden of care and the SOC of Alzheimer's caregivers by making several cognitive and behavioral changes, an effect that is justified by cognitive-behavioral theories. ${ }^{42}$

Overall, the findings of the present study confirm the application of CBTAC in reducing the burden of care and increasing the SOC of caregivers of Alzheimer's patients. Finally, it is necessary to state that the present study had some limitations. The limitations of the present study are lack of control over things such as caregivers' education, their necessary information about Alzheimer's disease, caregiver competence, closeness, and intimacy rate of caregiver with the patient.

For this reason, it is recommended that future studies be more controlled to clarify the impact of CBTAC on caregivers. Lack of attention to the gender of caregivers was one of the limitations. In this regard, it is recommended to consider in future studies whether the gender of caregivers is useful in the extent to which they are affected by the CBTAC protocol. Another limitation was the impossibility of the follow-up of the experiment after the study completed.

\section{Conclusion}

Based on the research results, the CBTAC is an effective intervention in decreasing caregiving burden and increasing the SOC of Alzheimer's caregivers. Therefore, CBTAC is a multicomponent intervention that can be

Table 4. Results of Univariate Analysis of Covariance on the Mean of Post-test Scores of Dependent Variables in 2 Experimental and Control Groups

\begin{tabular}{ccccccc}
\hline Variables & SS & $\boldsymbol{d f}$ & MS & F & Effect Value \\
\hline Burdens of Alzheimer's disease & 165.430 & 1 & 165.430 & 12.88 & 0.001 \\
SOC & 876.464 & 1 & 876.464 & 8.09 & 0.007 \\
\hline
\end{tabular}


used to improve the competency and mental health of Alzheimer's caregivers.

\section{Conflict of Interest Disclosure}

The authors declare that they have no conflict of interests.

\section{Ethical Statement}

All ethical considerations such as personal satisfaction, data retention and destruction, and informed participation have taken into account following the Declaration of Helsinki. ${ }^{46}$

\section{References}

1. Livingston G, Sommerlad A, Orgeta V, Costafreda SG, Huntley J, Ames D, et al. Dementia prevention, intervention, and care. Lancet. 2017;390(10113):2673-734. doi: 10.1016/S01406736(17)31363-6

2. Bondi MW, Edmonds EC, Salmon DP. Alzheimer's disease: past, present, and future. J Int Neuropsychol Soc. 2017;23(910):818-31. doi: 10.1017/S135561771700100X

3. Apostolova LG. Alzheimer Disease. Continuum. 2016;22(2 Dementia):419-34. doi: 10.1212/CON.0000000000000307

4. Peri A, Serio M. Neuroprotective effects of the Alzheimer's disease-related gene seladin-1. J Mol Endocrinol. 2008;41(5):251-61. doi: 10.1677/JME-08-0071

5. Cummings JL. Alzheimer's disease. N Engl J Med. 2004;351(1):56-67. doi: 10.1056/NEJMra040223

6. Alipour F, Oryan S, Sharifzadeh M, Karimzadeh F, Kafami $\mathrm{L}$, Irannejad $\mathrm{H}$, et al. The neuroprotective effect of a triazine derivative in an Alzheimer's rat model. Acta Med Iran. 2015;53(1):8-16.

7. Anand R, Gill KD, Mahdi AA. Therapeutics of Alzheimer's disease: Past, present and future. Neuropharmacology. 2014;76 (Pt A):27-50. doi: 10.1016/j.neuropharm.2013.07.004

8. Ramos Bernardes da Silva Filho S, Oliveira Barbosa JH, Rondinoni C, Dos Santos AC, Garrido Salmon CE, da Costa Lima NK, et al. Neuro-degeneration profile of Alzheimer's patients: A brain morphometry study. Neuroimage Clin. 2017;15:15-24. doi: 10.1016/j.nicl.2017.04.001

9. Boyd M. Behavioral disturbances associated with dementia: nursing implications. J Am Psychiatr Nurses Assoc. 2001;7(6):14-22 doi: 10.1067/mpn.2001.120878

10. Pudelewicz A, Talarska D, Bączyk G. Burden of caregivers of patients with Alzheimer's disease. Scand J Caring Sci. 2019;33(2):336-41. doi: 10.1111/scs.12626

11. Zucchella C, Sinforiani E, Tamburin S, Federico A, Mantovani E, Bernini $S$, et al. The multidisciplinary approach to Alzheimer's disease and dementia. A narrative review of nonpharmacological treatment. Front Neurol. 2018;9:1058. doi: 10.3389/fneur.2018.01058

12. Ferrara M, Langiano E, Di Brango T, De Vito E, Di Cioccio L, Bauco C. Prevalence of stress, anxiety and depression in with Alzheimer caregivers. Health Qual Life Outcomes. 2008;6:93. doi: 10.1186/1477-7525-6-93

13. Cole JC, Ito D, Chen YJ, Cheng R, Bolognese J, Li-McLeod J. Impact of Alzheimer's Disease on Caregiver Questionnaire: internal consistency, convergent validity, and test-retest reliability of a new measure for assessing caregiver burden. Health Qual Life Outcomes. 2014;12:114. doi: 10.1186/ s12955-014-0114-3

14. Adelman RD, Tmanova LL, Delgado D, Dion S, Lachs MS. Caregiver burden: a clinical review. JAMA. 2014;311(10):1052-60. doi: 10.1001/jama.2014.304
15. Volicer L. Caregiver burden in dementia care: Prevalence and health effects. Curr Psychiatry Rep. 2005;3(1):20-5. doi: 10.1007/BF02629405

16. Montgomery W, Goren A, Kahle-Wrobleski K, Nakamura T, Ueda K. Alzheimer's disease severity and its association with patient and caregiver quality of life in Japan: results of a community-based survey. BMC Geriatr. 2018;18(1):141. doi: 10.1186/s12877-018-0831-2

17. Sadik K, Wilcock G. The increasing burden of Alzheimer disease. Alzheimer Dis Assoc Disord. 2003;17 Suppl 3:S75-9.

18. Rymer S, Salloway S, Norton L, Malloy P, Correia S, Monast D. Impaired awareness, behavior disturbance, and caregiver burden in Alzheimer disease. Alzheimer Dis Assoc Disord. 2002;16(4):248-53.

19. Mohamed S, Rosenheck R, Lyketsos CG, Schneider LS. Caregiver burden in Alzheimer disease: cross-sectional and longitudinal patient correlates. Am J Geriatr Psychiatry. 2010;18(10):917-27.

20. Brodaty H, Woodward M, Boundy K, Ames D, Balshaw R. Prevalence and predictors of burden in caregivers of people with dementia. Am J Geriatr Psychiatry. 2014;22(8):756-65. doi: 10.1016/j.jagp.2013.05.004

21. Etters L, Goodall D, Harrison BE. Caregiver burden among dementia patient caregivers: a review of the literature. J Am Acad Nurse Pract. 2008;20(8):423-8. doi: 10.1111/j.17457599.2008.00342.x

22. Antonovsky A. Health, Stress, and Coping. San Francisco, CA, US: Jossey-Bass; 1979.

23. Antonovsky A. Unraveling the mystery of health: How people manage stress and stay well. San Francisco, CA, US: JosseyBass; 1987.

24. Eriksson $M$, Lindström B, Lilja J. A sense of coherence and health. Salutogenesis in a societal context: Aland, a special case? J Epidemiol Community Health. 2007;61(8):684-8. doi: 10.1136/jech.2006.047498

25. Andren S, Elmstahl S. The relationship between caregiver burden, caregivers' perceived health and their sense of coherence in caring for elders with dementia. J Clin Nurs. 2008;17(6):790-9. doi: 10.1111/j.1365-2702.2007.02066.x

26. Pretorius C, Walker S, Heyns PM. Sense of coherence amongst male caregivers in dementia: A South African perspective. Dementia. 2009;8(1):79-94. doi: 10.1177/1471301208099046

27. Potgieter JC, Heyns PM. Caring for a Spouse with Alzheimer's Disease: Stressors and Strengths. S Afr J Psychol. 2006;36(3):547-63. doi: 10.1177/008124630603600307

28. Elnasseh A, Trujillo M, Peralta S, Stolfi M, Morelli E, Perrin $P$, et al. Family dynamics and personal strengths among dementia caregivers in Argentina. Int J Alzheimers Dis. 2016;2016:2386728. doi: 10.1155/2016/2386728

29. Välimäki $T$, Martikainen J, Hongisto $K$, Fraunberg $M$, Hallikainen I, Sivenius J, et al. Decreasing sense of coherence and its determinants in spousal caregivers of persons with mild Alzheimer's disease in three year follow-up: ALSOVA study. Int Psychogeriatr. 2014;26(7):1211-20. doi: 10.1017/ S1041610214000428

30. Childers KM. Sense of Coherence and caregivers of persons with dementia. Behav Sci (Basel). 2019;9(2):14. doi: 10.3390/ bs9020014

31. Tomaskova H, Kuhnova J, Cimler R, Dolezal O, Kuca K. Prediction of population with Alzheimer's disease in the European Union using a system dynamics model. Neuropsychiatr Dis Treat. 2016;12:1589-98. doi: 10.2147/ 
NDT.S107969

32. Ferretti L, McCurry SM, Logsdon R, Gibbons L, Teri L. Anxiety and Alzheimer's disease. J Geriatr Psychiatry Neurol. 2001;14(1):52-8. doi: 10.1177/089198870101400111

33. Modrego PJ. Depression in Alzheimer's disease. Pathophysiology, diagnosis, and treatment. J Alzheimers Dis. 2010;21(4):1077-87.

34. Golden Z, Golden CJ. The differential impacts of Alzheimer's dementia, head injury, and stroke on personality dysfunction. Int J Neurosci. 2003;113(6):869-78. doi: 10.1080/00207450390200927

35. Brzecka A, Leszek J, Ashraf GM, Ejma M, Avila-Rodriguez MF, Yarla NS, et al. Sleep disorders associated with Alzheimer's disease: a perspective. Front Neurosci. 2018;12:330. doi: 10.3389/fnins.2018.00330

36. Kiyak HA, Teri L, Borson S. Physical and functional health assessment in normal aging and in Alzheimer's disease: selfreports vs family reports. Gerontologist. 1994;34(3):324-30.

37. Barnes RF, Raskind MA, Scott M, Murphy C. Problems of families caring for Alzheimer patients: use of a support group. J Am Geriatr Soc. 1981;29(2):80-5. doi: 10.1111/j.15325415.1981.tb01233.x

38. Maresova P, Mohelska H, Dolejs J, Kuca K. Socio-economic aspects of Alzheimer's disease. Curr Alzheimer Res. 2015;12(9):903-11.

39. Verulava T, Grdzelishvili A, Magaldadze M, Makharashvili A, Chibukhaia G, Jorbenadze $R$, et al. Social problems of Alzheimer Patients and their family members. Home Health Care Manag Pract. 2018;30(4):175-8. doi: 10.1177/1084822318775703

40. Molina F, Montoya-Rodríguez M. A Review of psychological intervention in Alzheimer's disease. International Journal of Psychology and Psychological Therapy. 2012;12:373-88.

41. Forstmeier S, Maercker A, Savaskan E, Roth T. Cognitive behavioural treatment for mild Alzheimer's patients and their caregivers (CBTAC): study protocol for a randomized controlled trial. Trials. 2015;16:526. doi: 10.1186/s13063015-1043-0

42. Kalodner CR. Cognitive-behavioral Theories. Counseling and Psychotherapy. 5th ed. Alexandria, VA, US: American Counseling Association; 2011. p. 193-213.

43. Antonovsky A. The structure and properties of the sense of coherence scale. Soc Sci Med. 1993;36(6):725-33. doi: 10.1016/0277-9536(93)90033-Z

44. Eriksson M, Lindstrom B. Validity of Antonovsky's sense of coherence scale: a systematic review. J Epidemiol Community Health. 2005;59(6):460-6. doi: 10.1136/jech.2003.018085

45. Alipour A, Sharif N. Validity and reliability of the Sense of Coherence (SOC) questionnaire in university students. Pajoohande. 2012;17(1):50-6.

46. World Medical Association Declaration of Helsinki: ethical principles for medical research involving human subjects. Jama. 2013;310(20):2191-4. doi: 10.1001/jama.2013.281053 